\title{
Restoring SIRT6 Expression in Hutchinson-Gilford Progeria Syndrome Cells Impedes Premature Senescence and Formation of Dysmorphic Nuclei
}

\author{
Helal Endisha ${ }^{a} \quad$ Jacqueline Merrill-Schools ${ }^{a} b \quad$ Min Zhao $^{a} \quad$ Molly Bristol $^{a}$ \\ Xu Wang ${ }^{a}$ Nard Kubben ${ }^{c}$ Lynne W. Elmore ${ }^{a, b}$ \\ ${ }^{a}$ Department of Pathology and b Massey Cancer Center, Virginia Commonwealth University, Richmond, Va., and \\ 'National Cancer Institute, National Institutes of Health, Bethesda, Md., USA
}

\section{Key Words}

Hutchinson-Gilford progeria syndrome · SIRT6 ·

Senescence $\cdot$ Anti-aging $\cdot$ Progerin

\begin{abstract}
Objectives: Mice overexpressing SIRT6 live longer than wildtype mice while SIRT6 knockout mice exhibit similar degenerative phenotypes as individuals with Hutchinson-Gilford progeria syndrome (HGPS). Thus, we sought to test whether levels of SIRT6 are reduced in cells from individuals with HGPS and whether restored SIRT6 expression may impede premature aging phenotypes. Methods: Levels of endogenous SIRT6 and progerin in HGPS and normal fibroblasts were assessed by Western blotting and immunofluorescence. A tetracycline-inducible system was utilized to test whether progerin causes a rapid reduction in SIRT6 protein. SIRT6 was overexpressed in HGPS cells via lentiviral infection with biological endpoints including senescence-associated $\beta$-galactosidase (SA- $\beta$-gal) positivity, frequency of nuclear atypia, the number of 53BP1-positive DNA damage foci and growth rates. Results: Typical HGPS fibroblasts express lower levels of SIRT6 than fibroblasts from normal and atypical HGPS donors. Experimental induction of progerin did not cause a detectable reduction of SIRT6 protein. However,
\end{abstract}

overexpression of SIRT6 in HGPS cells was associated with a reduced frequency of SA- $\beta$-gal positivity, fewer misshapen nuclei, fewer DNA damage foci, and increased growth rates. Conclusions: Typical HGPS fibroblasts exhibit reduced levels of SIRT6 protein via a mechanism that remains to be elucidated. Our findings suggest that restoring SIRT6 expression in HGPS cells may partially impede senescence and the formation of dysmorphic nuclei.

(c) 2015 S. Karger AG, Basel

\section{Introduction}

Hutchinson-Gilford progeria syndrome (HGPS) is a rare premature aging syndrome that typically arises from a de novo single nucleotide mutation $(1824 \mathrm{C} \rightarrow \mathrm{T})$ in the LMNA gene $[1,2]$. As a result, in 'typical' HGPS cells the mutated lamin A protein (progerin) remains farnesylated and permanently attached to the nuclear membrane [3]. Experimental data indicate that when progerin accumulates in cells, nuclear membranes become deformed, telo-

H.E. and J.M.-S. share joint first authorship due to their equally significant contributions to the study.

\section{KARGER 125}

(c) 2015 S. Karger AG, Base

$1015-2008 / 15 / 0821-0009 \$ 39.50 / 0$

E-Mail karger@karger.com

www.karger.com/pat
L.W. Elmore

Department of Pathology, Virginia Commonwealth University

1101 East Marshall Street

Richmond, VA 23298 (USA)

E-Mail lelmore@mcvh-vcu.edu 
Table 1. Normal and HGPS human fibroblast cell strains

\begin{tabular}{llllc}
\hline Cell strain & Diagnosis & Cell type & Sex & $\begin{array}{l}\text { Donor } \\
\text { age, } \\
\text { years }\end{array}$ \\
\hline AG01972 & typical HGPS & dermal fibroblast & female & 14 \\
AG11513 & typical HGPS & dermal fibroblast & female & 8 \\
AG03199 & typical HGPS & dermal fibroblast & female & 10 \\
AG07493 & atypical HGPS & dermal fibroblast & female & 2 \\
GM00969 & normal & $\begin{array}{l}\text { dermal fibroblast } \\
\text { dermal female }\end{array}$ & 2 \\
AG09602 & normal & femalle & 92 \\
\hline
\end{tabular}

meres become dysfunctional, DNA damage remains unrepaired, and cells enter a permanently arrested growth state termed cellular senescence $[4,5]$. It has been postulated that premature aging at the cellular level collectively causes disruption of tissue homeostasis and compromised tissue/organ function, possibly contributing to the manifestation of drastically accelerated aging conditions (i.e. alopecia, loss of subcutaneous fat, progressive joint contractures, bone changes, atherosclerosis and stroke) in children with HGPS [6].

Cellular and organismal aging are accompanied by epigenetic alterations, including DNA methylation changes, posttranslational modification of histones residues, and altered gene expression due to the loss of heterochromatic chromatin in telomeric and subtelomeric regions $[7,8]$. Some of the initial data linking aging and epigenetic regulation were generated in lower eukaryotic model systems. Extra copies of the silent information regulator-2 (Sir2) gene, which encodes an NAD-dependent histone deacetylase, increases the replicative life span in Saccharomyces cerevisiae, Caenorhabditis elegans and Drosophila melanogaster [9]. Conversely, S. cerevisiae with mutations in Sir2 exhibits increased genomic instability and a shortened replicative life span [10]. In mammals, there are seven Sir2 family members (SIRT1-7) [11], and data are accumulating to indicate that SIRT6 may be the sirtuin with particular relevance for regulating life span and preserving genomic integrity in mammals [12]. SIRT6 knockout mice $\left(\right.$ SIRT6 $^{-I-}$ ) show no abnormalities at birth apart from a reduced body size shortly after birth [13]. However, mice at 3 weeks of age exhibited degenerative phenotypes including loss of subcutaneous fat, lordokyphosis, colitis, severe lymphopenia and a general 'failure to thrive'. The mice ultimately died at postnatal day 24 , exhibiting many premature aging phenotypes. On the contrary, male transgenic mice overex- pressing SIRT6 have a significantly longer life span than wild-type control mice [14]. These intriguing observations, together with the knowledge that SIRT6 associates specifically with telomeres $[15,16]$ and its depletion in normal human cells leads to telomere dysfunction and premature aging [17], prompted us to investigate whether SIRT6 expression may be reduced in HGPS cells and, if so, whether restoring SIRT6 expression in these senescent-prone cells may confer antiaging effects.

\section{Materials and Methods}

\section{Cell Cultures}

Three strains of typical HGPS dermal fibroblasts, one strain of atypical HGPS dermal fibroblasts, and two strains of normal dermal fibroblasts were purchased from the Coriell Institute for Medical Research (table 1). Normal dermal fibroblasts from a young donor ( 2 years old) and an old donor (92 years old) were included in the cell panel for two reasons: (1) published data suggest that progerin accumulates in cells during the normal aging process [18, 19] and (2) we believed that including cells from normal donors of such disparate ages could provide insights into whether physiologically aged cells from young donors were more similar to cells from donors that are chronologically young or old in terms of SIRT6 expression. Mycoplasma-negative cells (LookOut ${ }^{\circledR}$ Mycoplasma PCR Detection Kit; Sigma) were grown at $37^{\circ} \mathrm{C}$ in $5 \% \mathrm{CO}_{2}$ in Eagle's minimum essential medium with Earle's salts (Cellgro) supplemented with $15 \%(\mathrm{v} / \mathrm{v})$ fetal bovine serum (Invitrogen), MEM nonessential amino acids (Cellgro), $200 \mathrm{~mm} \mathrm{~L}$-glutamine (Gibco) and antibiotic/antimycotic (Invitrogen). Population doubling (PD) was calculated from cell counts taken at each passage using the formula: $\mathrm{PD}=\left[\log \left(\mathrm{N} / \mathrm{X}_{\mathrm{o}}\right)\right] / \log 2$, where $\mathrm{N}$ is the final cell count and $\mathrm{X}_{\mathrm{O}}$ is the initial cell count.

\section{Lentiviral Infections and Constructs}

SIRT6 and empty vector lentiviral supernatants were generated in the Biological Macromolecule Shared Resource Core at Virginia Commonwealth University. The SIRT6 gene was cloned in a pcDNA3.1+ vector backbone (Addgene; plasmid 13,817) and then expressed in the pCDH-CMV-MCS-EF1-Puro expression lentivector (System Biosciences). Typical AG11513 and AG01972 HGPS cells were each seeded in triplicate in $60-\mathrm{mm}$ dishes at a density of $2 \times 10^{5}$ cells per dish. For each cell strain, a plate of uninfected cells was included as a control. Cells were infected at a multiplicity of infection of one. Polybrene $(8 \mu \mathrm{g} / \mathrm{ml}$; Santa Cruz Biotechnology) was added to the diluted viral supernatant to increase transduction efficiency. Cells were infected overnight, with fresh media being added the next day. Following a 24-hour recovery phase, cells were exposed to $0.7 \mu \mathrm{g} / \mathrm{ml}$ of puromycin (Sigma) for 4-5 days to remove the uninfected cells.

We established a 'TET-on' progerin expression in hTERT-immortalized human dermal fibroblasts. N-terminally EGFP-tagged progerin was recloned from a previously described pBabe puro EGFP-progerin plasmid [20] into a pENTR1A no ccDB vector (Addgene; plasmid w48-1), which was subsequently used to transfer the EGFP-progerin sequence into a pLenti CMV TRE3G Neo
10

Pathobiology 2015;82:9-20 DOI: $10.1159 / 000368856$
Endisha/Merrill-Schools/Zhao/Bristol/ Wang/Kubben/Elmore 
plasmid (Addgene; plasmid w813-1) by Gateway-mediated LR recombination according to the manufacturer's instructions (Invitrogen). Next, hTERT immortalized human dermal fibroblasts were twice infected with lentivirus from the generated pLenti CMV TRE3G Neo EGFP-progerin plasmid as well as the pLenti CMV rtTA3 Hygro plasmid (Addgene; plasmid w785-1), which expresses the tetracycline repressor A3 mutant. Infected cells were selected by antibiotics ( $200 \mu \mathrm{g} / \mathrm{ml}$ of G418 and $200 \mu \mathrm{g} / \mathrm{ml}$ of hygromycin). Individual clones were grown by serial dilution and selected on having expression levels of EGFP-progerin comparable to endogenous lamin A after a 4-day induction with $1,000 \mathrm{ng} / \mathrm{ml}$ of doxycycline. These fibroblasts were plated with the same medium used for normal and progeria fibroblasts (described above) supplemented with $750 \mathrm{ng} / \mathrm{ml}$ of doxycycline for up to 12 days to induce expression of the EGFP-progerin fusion protein.

\section{Western Blotting}

Fibroblasts were lysed in RIPA buffer ( 25 mM Tris- $\mathrm{HCl}, \mathrm{pH} 7.6$, $150 \mathrm{~mm} \mathrm{NaCl}, 1 \% \mathrm{NP}-40,1 \%$ sodium deoxycholate, $0.1 \%$ sodium dodecyl sulfate) containing Halt Protease and Phosphatase Inhibitor Cocktail (Thermo Scientific). Protein concentrations were quantified using the BioRad Protein Assay (BioRad Laboratories) and $30 \mu \mathrm{g}$ of protein were run per lane on an $8-10 \%$ sodium dodecyl sulfate polyacrylamide gel. After electrotransferring the protein from the gel to a nitrocellulose membrane, the membrane was blocked for $1 \mathrm{~h}$ at room temperature followed by overnight incubation with diluted primary antibodies: rabbit anti-SIRT6 (No. 2590; Cell Signaling Technology); mouse anti-progerin antibody (ab66587; Abcam) or mouse monoclonal anti- $\beta$-actin (sc-47778; Santa Cruz Biotechnology), as recommended by the manufacturers. Depending on the derivation of the respective primary antibodies, either an anti-mouse or anti-rabbit horseradish peroxidase-conjugated secondary antibody (1:5,000 dilution) was used followed by SuperSignal ${ }^{\circledR}$ West Pico Chemiluminescent Substrate (Thermo Scientific) to detect signals. SIRT6 gel quantification was determined using ImageJ (a Java-based program developed at the National Institutes of Health). Each sample was normalized with actin and area values were presented relative to GM00969 normal fibroblasts.

\section{SIRT6 and 53BP1 Immunolabeling}

Immunofluorescence was utilized to assess the relative expression levels and cellular localization of SIRT6. This involved a 10min fixation in $4 \%$ paraformaldehyde, a $0.1 \%$ Triton-X permeabilization step for $10 \mathrm{~min}$, and then blocking in phosphate buffer containing 3\% fetal bovine serum for $20 \mathrm{~min}$. Cells were then incubated overnight with rabbit anti-human SIRT6 at $10 \mu \mathrm{g} / \mathrm{ml}$ (No. 2590; Cell Signaling Technology) at $4^{\circ} \mathrm{C}$, followed by a 45 min incubation at room temperature with Alexa goat anti-rabbit $488(4 \mu \mathrm{g} / \mathrm{ml}) .4^{\prime}, 6$-Diamidino-2-phenylindole, dihydrochloride (DAPI; $1.5 \mu \mathrm{g} / \mathrm{ml}$ ) was added to VECTASHIELD ${ }^{\circledR}$ antifade mounting media (Vector Laboratories) to detect nuclei. The same general fixation and staining protocols were followed for 53BP1 immunolabeling with the exception of the use of a 53BP1 monoclonal antibody at $0.8 \mu \mathrm{g} / \mathrm{ml}$ (No. 612523BD; Transduction Laboratories), followed by incubation with Alexa goat anti-mouse 568 . For each normal and HGPS cell strain, primary antibody was omitted as a negative control. For determination of the average number of 53BP1-positive DNA damage foci per nuclei, three independent experiments were conducted with each involving the scoring of
100 nuclei for each sample using Image J with a uniform threshold value setting for all samples.

\section{Cellular Growth Rates}

Initially, we compared the in vitro life span of HGPS versus normal dermal fibroblasts by determining their respective PD time following serial passaging in culture. Growth rates were then compared in the short term as follows: in a 12-well plate, each sample was seeded in triplicate for 3 time points; after 24,48 and $72 \mathrm{~h}$, the respective triplicate wells were trypsinized and the doubling times calculated using Doubling Time software (http://www.doublingtime.com); the average doubling times and standard error from each replicate were calculated and presented graphically.

\section{Morphometric Analysis}

The presence of progerin adversely affects the integrity of the nuclear lamina, resulting in misshapen nuclei and nuclear blebs [6]. To directly test whether restoration of SIRT6 improves nuclear scaffolding defects so characteristic of HGPS cells, we measured the perimeter and area of 100-200 randomly selected DAPIstained nuclei of dermal fibroblasts from 2 normal donors at PD 20, HGPS AG11513 at low and high passage, and HGPS AG11513 + SIRT6 at high passage using ImageJ. The nuclear roundness or contour ratio $\left(4 \pi \times\right.$ area/perimeter $\left.{ }^{2}\right)$ was then determined with a value $<0.75$ being scored as abnormal (the contour ratio of a circle is 1) $[21,22]$.

Senescence-Associated $\beta$-Galactosidase Histochemical Staining

To assess cellular senescence, a standard senescence-associated $\beta$-galactosidase (SA- $\beta$-gal) histochemical staining was performed using the kit from Cell Signaling Technology as described previously [23]. Senescence was evaluated in HGPS cells over time by counting the number of cells that exhibited a flattened and enlarged cell morphology together with intense cytoplasmic SA- $\beta$ gal staining in 3 fields for each replicate.

\section{Statistical Analysis}

Cell growth, contour ratio, SA- $\beta$-gal frequency and immunoblotting densitometry differences were analyzed using two-tailed Student's t test and $\mathrm{p}<0.05$ was considered significant.

\section{Results}

We assessed the levels of endogenous SIRT6 in a panel of normal and HGPS fibroblasts (table 1), and found that there were significantly $(\mathrm{p}=0.02)$ reduced levels of SIRT6 protein in typical HGPS cells $(0.23 \pm 0.19)$ when compared to normal fibroblasts $(1.3 \pm 0.42$; fig. $1 \mathrm{a})$. We included one atypical HGPS cell strain (AG07493) in the panel, which, due to a different mutation in the lamin $\mathrm{A}$ gene, does not express progerin and consequently is associated with a less severe progeroid syndrome [24, 25]. The relative level of SIRT6 protein in this atypical cell strain (1.1) was in the range of the normal fibroblasts (1.0-1.6) rather than those densitometric values gener- 


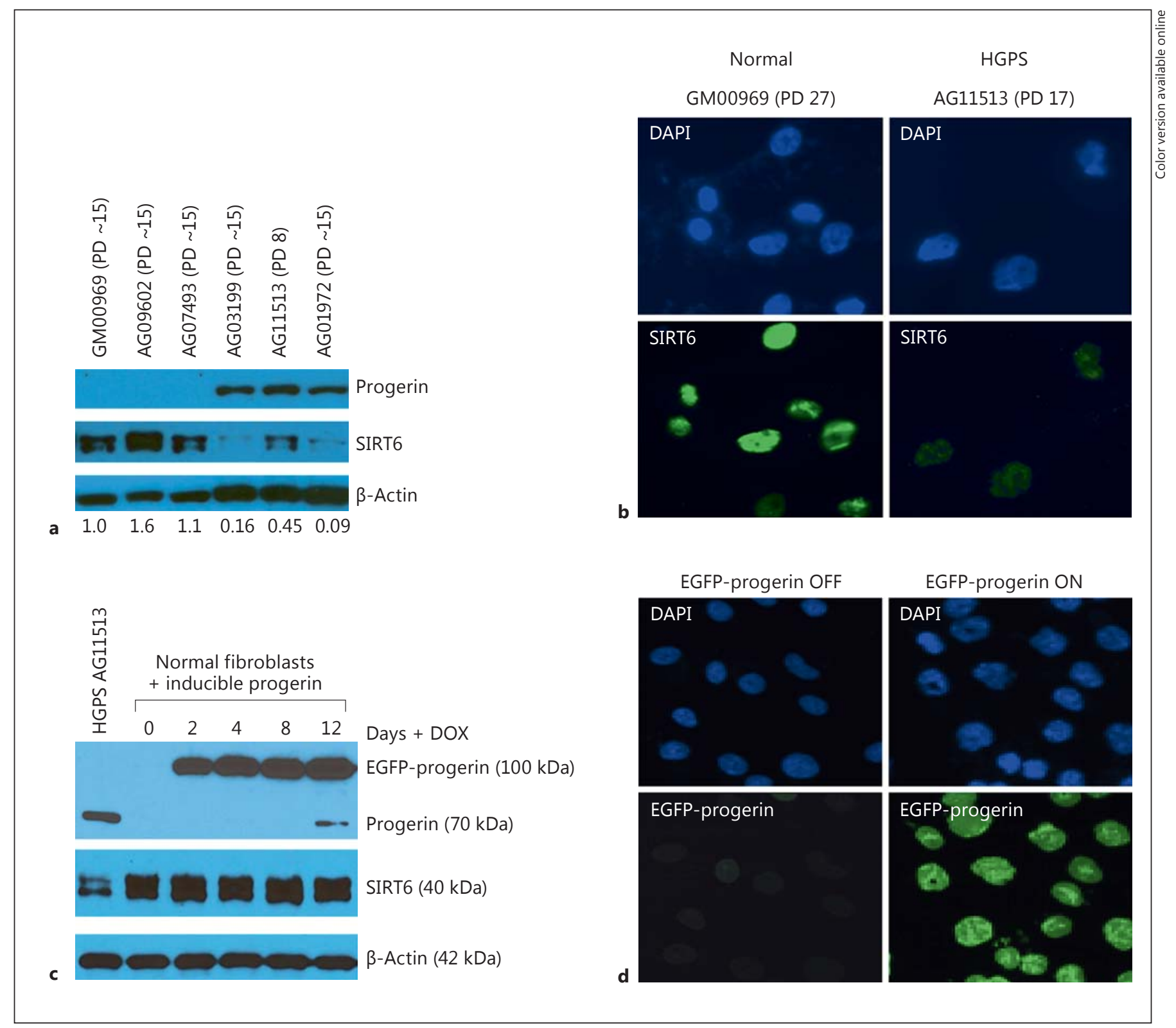

Fig. 1. Reduced expression of SIRT6 in typical HGPS cells. a Western blotting demonstrating that only typical HGPS cells express detectable levels of progerin with significantly reduced SIRT6 in progerin-expressing HGPS human cells relative to normal fibroblasts $(p=0.02)$. Values below the blot represent actin-normalized SIRT6 densitometric values relative to GM00969 normal fibroblasts. b Immunofluorescence confirms reduced expression of SIRT6 in typical HGPS cells relative to dermal fibroblasts from a normal donor. No primary antibody controls imparted undetectable fluorescent signal (data not shown). c Normal dermal fibroblasts \pm doxycycline-inducible progerin express equally strong lev- els of SIRT6. AG11513 cells (lane 1) were included as a positive control for endogenous progerin, a negative control for exogenous progerin, and for further confirmation that typical HGPS cells express lower levels of SIRT6 than uninduced normal dermal fibroblasts (lane 2). After 12 days of sustained high levels of exogenous progerin, a $\sim 70-\mathrm{kDa}$ protein was detectable, which may represent endogenous progerin. d Immunofluorescence confirms expression and correct nuclear localization of EGFP-progerin in the TET-on inducible progerin cell line after 4 days of induction with doxycycline. 
ated for the typical HGPS cells (0.09-0.45). We did not observe detectable progerin by Western blotting in the atypical HGPS cells or low-passage normal fibroblasts. Highly consistent with our immunoblotting results, endogenous SIRT6 immunofluorescent intensity was much greater in normal dermal fibroblasts than typical HGPS fibroblasts (fig. 1b) and, as previously described [26], localized exclusively to nuclei.

Our finding of reduced SIRT6 expression in typical HGPS cells begs the question of whether progerin directly reduces expression of SIRT6. To test this possibility, we established a strain of human fibroblasts with a tightly regulated, doxycycline-inducible N-terminally EGFPtagged progerin protein. As shown in figure $1 \mathrm{c}$, there is undetectable endogenous or exogenous progerin protein expression in uninduced normal human fibroblasts. Addition of doxycycline to the culture media resulted in abundant expression of the EGFP-progerin fusion protein after 2 days, with further accumulation at 4,8 and 12 days. There was no evidence of reduced SIRT6 expression up to 12 days of ectopic progerin expression, suggesting that progerin does not directly cause reduction of SIRT6 protein expression. Investigating the SIRT6 protein status beyond 12 days of progerin induction was not feasible due to severe growth impairment and cell death caused by such prolonged ectopic progerin expression. AG11513 progeria cells were included as a positive control for endogenous progerin (fig. 1c, lane 1, 70-kDa band). Consistent with the data of figure 1a, the levels of SIRT6 in the progeria cells (fig. 1c) were much lower than those observed in normal cells in the uninduced state (fig. 1c, lane 2 ). Twelve days after the induction of exogenous progerin, a band comparable to the size of endogenous progerin was detected by Western blotting (fig. 1c, far right lane). It remains to be determined whether sustained high levels of ectopic progerin in normal human fibroblasts exhibiting severe cytotoxicity favors alternate splicing of the LMNA gene leading to progerin expression [5]. Immunolabeling for progerin confirmed expression and correct nuclear localization of EGFP-progerin in the TET-on inducible progerin cell line after 4 days of induction with doxycycline (fig. 1d).

We next tested whether restoring SIRT6 expression impedes the characteristic premature cellular aging of HGPS cells upon their serial passaging [27, 28]. Prior to establishing mass cultures of HGPS fibroblast stably overexpressing SIRT6, we assessed the proliferative capacity and frequency of SA- $\beta$-gal activity in 2 strains of typical HGPS cells over time. Highly consistently with previous reports [27, 28], we found that AG01972 and AG11513
HGPS cells had a much more limited proliferative potential than GM00969 and AG09602, two strains of dermal fibroblasts from normal donors. We have also shown that normal dermal fibroblasts from the 92-year-old donor had longer PD times than dermal fibroblasts from a 2 -year-old donor, with both senescing after 250 days in culture (fig. 2a). We confirmed with SA- $\beta$-gal histochemical staining that the limited proliferative potential of typical HGPS fibroblasts was due to widespread cellular senescence (fig. 2b, c).

Due to the characteristic long doubling times of HGPS fibroblasts, we used a lentiviral construct to stably overexpress SIRT6. After 4 days of puromycin selection, drugresistant HGPS cells (morphologically indistinguishable from the uninfected parental control; fig. 3a, middle panel vs. left panel) started repopulating the culture. In contrast, cultures that were infected with the empty lentiviral vector never recovered from puromycin selection (fig. 3a, left panel shows numerous unhealthy and dying cells). Repeat infections with 2 different typical HGPS cell strains resulted in the same inability to establish empty vector mass controls (data not shown), necessitating the use of the respective uninfected cell strains as baseline controls. Western blotting confirmed that the puromycin-resistant mass cultures from dermal fibroblasts from 2 different HGPS donors overexpressed SIRT6 (fig. 3b). Due to the high levels of SIRT6 overexpression (and, therefore, short exposure time), we did not detect the low levels of endogenous SIRT6 in either of the uninfected HGPS cell strains by immunoblotting. Immunofluorescence confirmed SIRT6 overexpression in infected HGPS cells and revealed that ectopically expressed SIRT6, like its endogenous counterpart, is localized to the nucleus (fig. 3c).

Our inability to establish drug-resistant empty vector controls coupled with the ease of establishing SIRT6 overexpressing HGPS cells preliminarily suggested that ectopic expression of SIRT6 provided a growth advantage to typical HGPS fibroblasts. To provide further evidence in support of this possibility, we compared the PD times of SIRT6 overexpressing AG11513 cells and uninfected parental cells at two (PD-matched) time points. We observed a statistically significant $(\mathrm{p}<0.04)$ reduction in $\mathrm{PD}$ times in HGPS/SIRT6 fibroblasts compared to uninfected cultures (fig. 4a). Reduced doubling times were observed shortly after the establishment of the SIRT6-overexpressing HGPS cells (i.e. PD 12) as well as at PD 20, when the vast majority of HGPS fibroblasts typically undergo cellular senescence. SA- $\beta$-gal histochemical staining revealed that HGPS/SIRT6 cells at PD 26, a doubling time that we could not achieve with uninfected HGPS 
Fig. 2. Typical HGPS cells undergo premature replicative senescence. a PD times were calculated in cultures of dermal fibroblasts from normal donors and individuals with HGPS. The two progeria cell strains (AG01972 and AG11513) were unable to be propagated beyond 45 and 90 days in culture, respectively. In contrast, two cell strains from normal donors (GM00969 and AG09602) continued to replicate in vitro for over 250 days. b Representative SA$\beta$-gal histochemical staining images showing widespread positivity and enlarged flattened cellular morphology in typical HGPS cells at PD 20 (right panel). At a similar PD, fibroblast cultures from a normal donor only occasionally revealed SA- $\beta$-galstained cells, with the vast majority of the cells displaying a more youthful, fusiform morphology (left panel). c Quantitative data revealing a time-dependent increase in SA- $\beta$-gal positivity in AG11513 HGPS cells. Asterisks indicate a statistically significant increase relative to AG11513 cells at PD $12(\mathrm{p}<0.012)$.
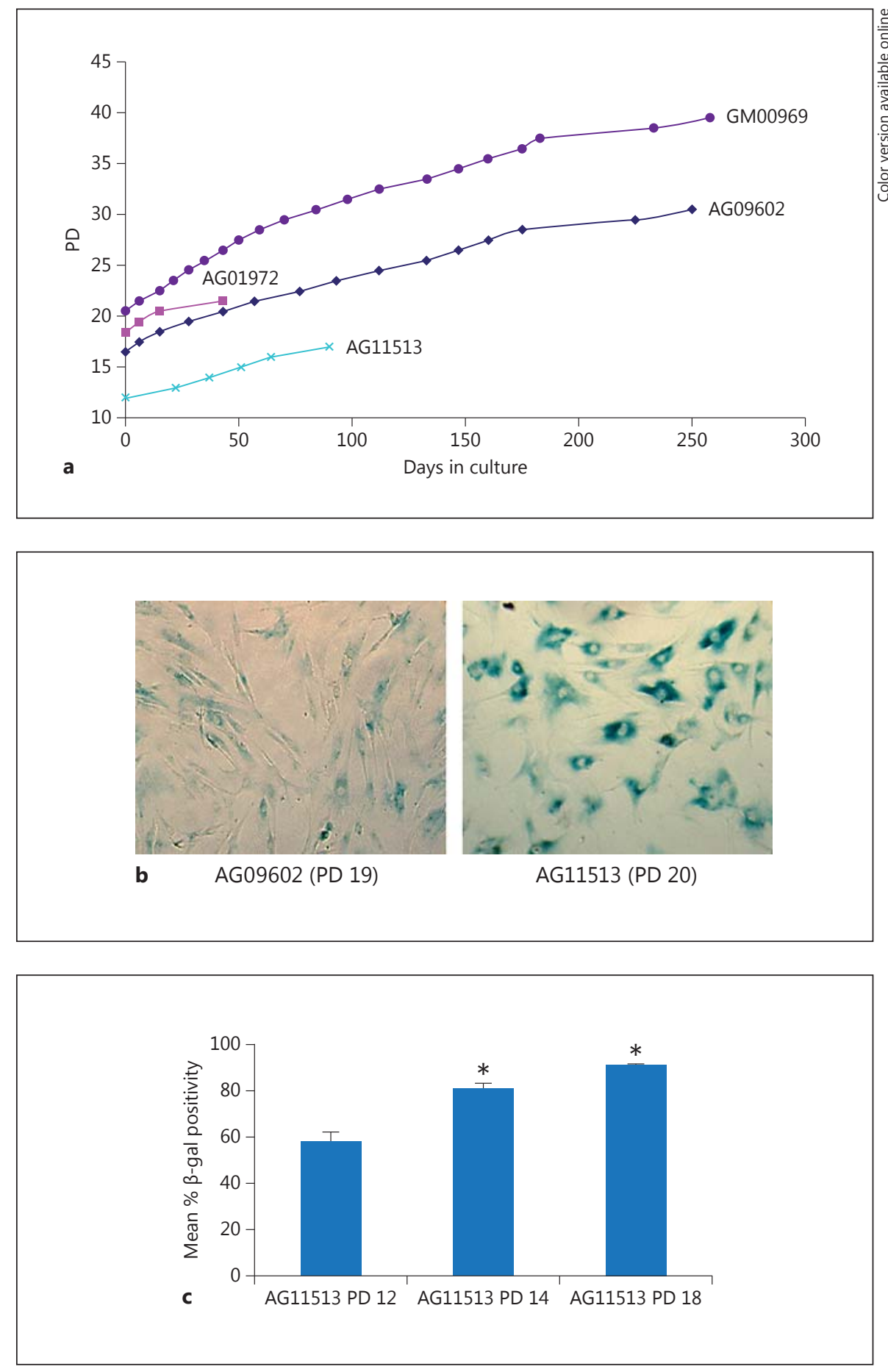

cells, exhibited a statistically significant reduction in the frequency of cellular senescence when compared to uninfected AG11513 cells at PD $12(\mathrm{p}=0.033)$ and at PD 20 $(\mathrm{p}=0.003$; fig. $4 \mathrm{~b})$. It was also evident in the light microscopic images (all at $\times 20$ ) that the SIRT6-infected proge- ria cells displayed a morphology (fig. 4c, right panel) more akin to low-passage progeria cells (fig. 4c, left panel displaying a spindle shape) than uninfected high-passage progeria cells with their enlarged and flattened appearance (middle panel; fig. 4c). 


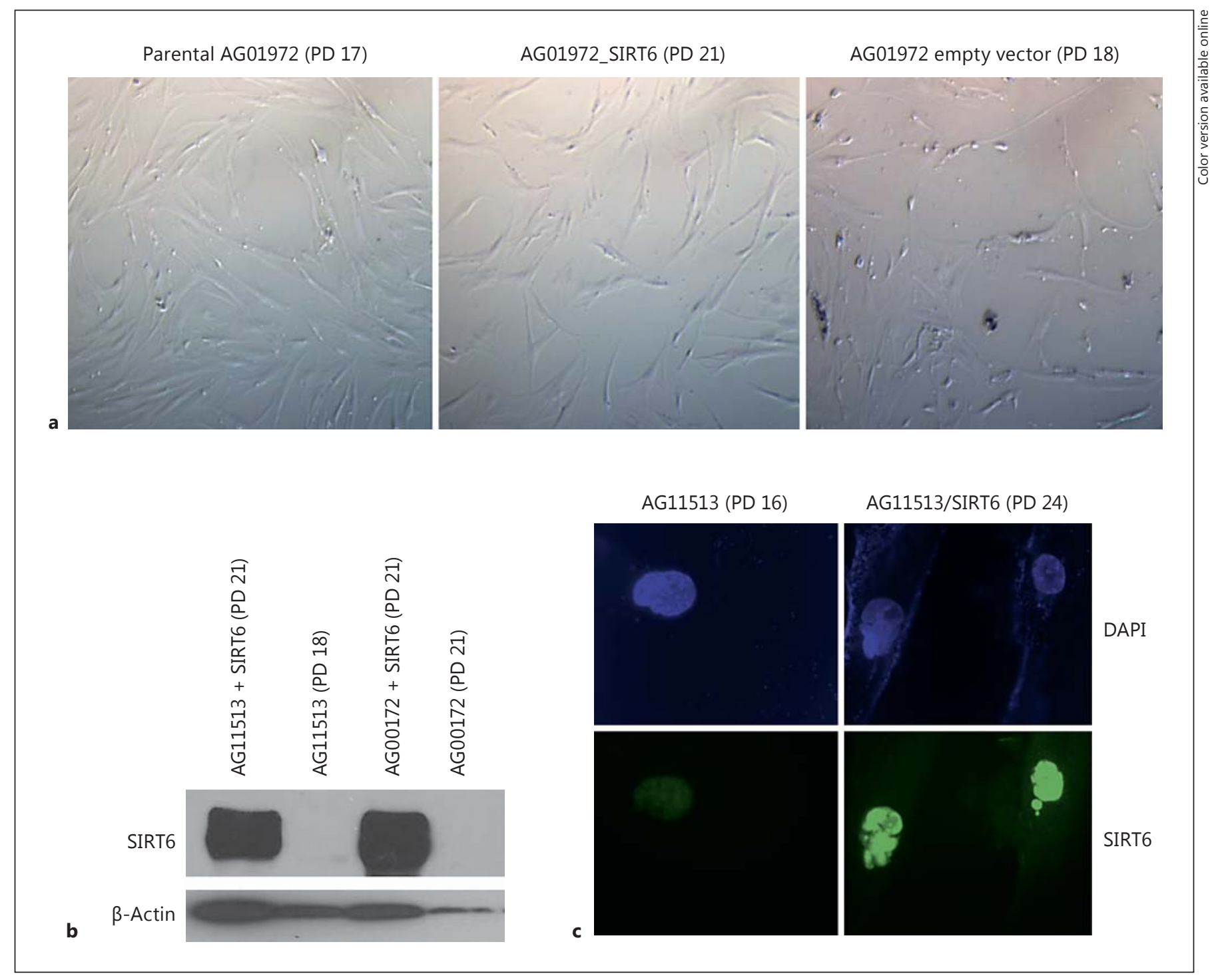

Fig. 3. Stable overexpression of SIRT6 in typical HGPS cells. a Following infection with SIRT6 retroviral supernatant, puromycinresistant cells populated the culture (middle panel showing AG01972 HGPS cells as a representative culture) whereas cells infected with viral supernatant from the empty vector control never recovered from drug selection (right panel). Uninfected parental AG01972 cells are included as a reference (left panel). Original magnification $\times 20$. b Western blot demonstrating high levels of exogenous SIRT6 overexpression in two typical HGPS cell strains. Due to the short exposure time, the low levels of endogenous
SIRT6 in typical HGPS cells were undetectable. $\beta$-Actin was included as a loading control. c SIRT6 immunolabeling to confirm low levels of endogenous SIRT6 in the uninfected controls (lower left panel) and strong nuclear expression of exogenous SIRT6 (lower right panel). DAPI-stained images (upper panels) allow for identification of all cells in the microscopic field and an assessment of nuclear morphology. Original magnification $\times 100$. No primary antibody controls imparted undetectable fluorescent signal (data not shown).
In addition to the onset of an accelerated cellular aging phenotype, typical HGPS cells also exhibit numerous changes in nuclear architecture, including lobulation of the nuclear envelope upon serial passaging [29]. Therefore, we investigated whether ectopic expression of SIRT6 could impede the progressive nuclear atypia in HGPS cells. We measured the nuclear area and perimeter using ImageJ, which then allowed for the determination of contour ratios in normal dermal fibroblasts, HGPS cells, and HGPS cells stably expressing SIRT6. For each culture, we 

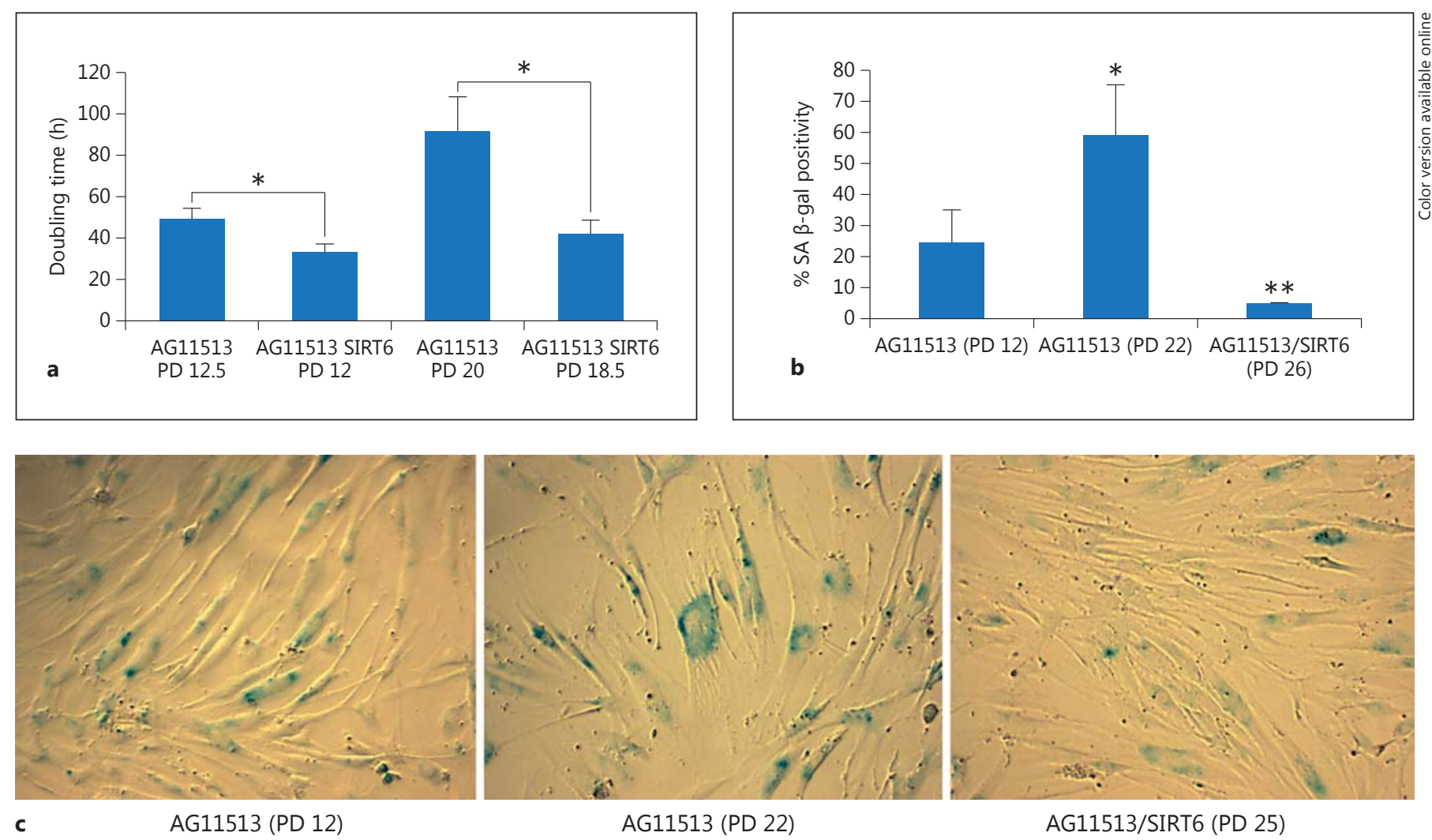

Fig. 4. HGPS cells ectopically expressing SIRT6 display an increased growth rate and a reduced frequency of SA- $\beta$-gal positivity. PD times of AG11513 HGPS fibroblasts \pm ectopic SIRT6 expression (a) and the frequency of SA- $\beta$-gal positivity in the AG11513 \pm SIRT6 (b). Bars represent mean \pm SD from 3 random

microscopic fields. Asterisks denote a statistically significant difference from low-passage AG11513 cells ( $\left.{ }^{*} \mathrm{p}=0.033 ;{ }^{* *} \mathrm{p}=0.003\right)$. c Representative light microscopic images of AG11513 HGPS cells histochemically stained for SA- $\beta$-gal following serial passaging in vitro. Original magnification $\times 20$.

determined the mean contour ratios as well as the frequency of abnormal nuclei, as defined by a single nucleus having a contour ratio of $<0.75$. As described previously [29], during early passaging the nuclei of HGPS cells appear normal with respect to their overall morphological features (fig. 5a, upper right panel compared to normal fibroblasts in the upper left panel). Our quantitative data confirmed these impressions: the mean contour ratios and the frequency of abnormal nuclei of low-passage HGPS cells were not different to values generated from fibroblasts from normal donors (fig. 5b). It is noteworthy that the number of abnormal nuclei in the normal fibroblasts from the nonagenarian was much more variable than normal and HGPS cells from young donors (fig. 5b). When AG11513 HGPS cells reached PD 18, they clearly exhibited enlarged nuclei with varying degrees of lobulation (fig. 5a, lower left panel) and, as expected, a statistically significant increase in the frequency of abnormal nuclei when compared to all other cultures ( $\mathrm{p}<0.0006$; fig. 5b). In contrast, HGPS cells with restored SIRT6 expression displayed nuclear features more reminiscent of low-passage HGPS cells (fig. 5a, lower right panel compared to low-passage HGPS cells in the upper right panel) and a statistically significant reduction in the frequency of abnormal nuclei when compared to high-passage HGPS cells ( $7.4 \pm 2.0$ vs. $23.8 \pm 2.1 ; \mathrm{p}=0.015)$. While the HGPS cells ectopically expressing SIRT6 displayed improved nuclear architecture, the frequency of abnormal nuclei was still statistically higher than that measured in low-passage HGPS cells $(7.4 \pm 2.0$ vs. $2.7 \pm 0.01$; $\mathrm{p}=$ $0.0006)$, indicating a partial rescue of this phenotype. The mean contour ratio for AG11513 ectopically expressing SIRT6 cells at PD 22 trended towards values observed in the normal dermal fibroblasts, but there were no statistically significant differences in contour ratios between normal and HGPS cells (young normal donor: $0.834 \pm$ 
Fig. 5. Ectopic expression of SIRT6 partially restores normal nuclear morphology in HGPS cells. a Representative DAPI-stained nuclei of dermal fibroblasts derived from a normal donor (97 years old, upper left panel; 2 years old, not shown), AG11513 HGPS cells at low $\mathrm{PD}$ (upper right panel) and high PD (lower left panel), and AG11513 + SIRT6 cells at PD 22 (lower right panel). b Morphological abnormalities in the nucleus of control and HGPS cells were quantified based on the contour ratio and the frequency of abnormal nuclei as defined by a contour ratio of $<0.75$. Superscript letters denote the following statistically significant differences. ${ }^{a}$ The mean number of abnormal nuclei in high-passage HGPS cells was statistically different from all other values $(p<0.0006) .{ }^{b}$ The mean number of abnormal nuclei in HGPS cells with restoration of SIRT6 expression was significantly lower than of high-passage HGPS cells $(23.8 \pm 2.1$ vs. $7.4 \pm 2.0 ; \mathrm{p}=0.0006)$. This value of $7.4 \pm 2.0$ was still significantly higher than low-passage HGPS cells (2.7 \pm $0.01 ; \mathrm{p}=0.015)$, indicating only partial improvement in nuclear atypia. The average contour ratios for HGPS cells ectopically expressing SIRT6 trended towards a more normal nuclear morphology, but the values did not reach statistical significance.
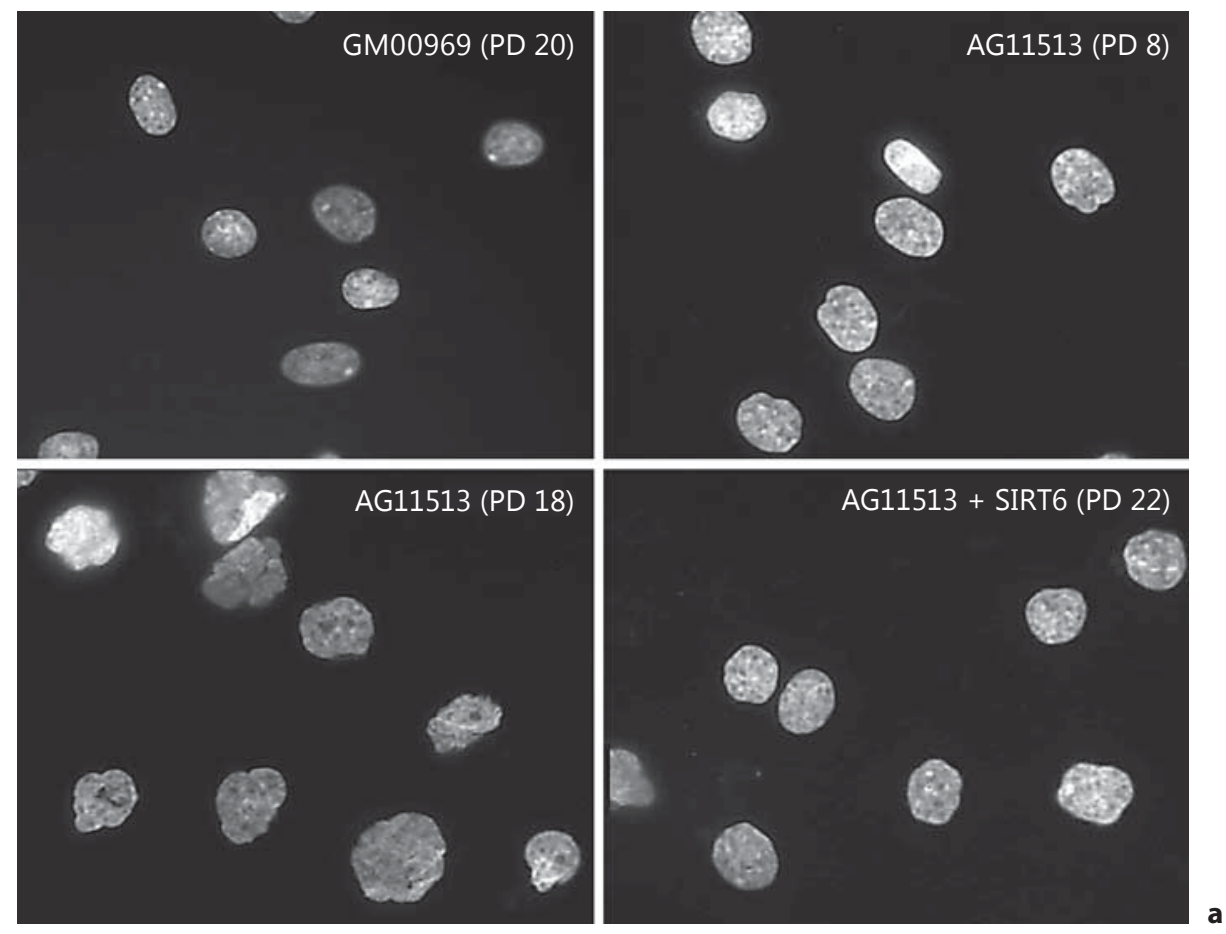

\begin{tabular}{|lrclc|}
\hline Cell strain & PD & $\begin{array}{l}\text { Donor age, } \\
\text { years }\end{array}$ & $\begin{array}{l}\text { Contour } \\
\text { ratio }\end{array}$ & $\begin{array}{l}\text { Abnormal } \\
\text { nuclei, \% }\end{array}$ \\
\hline GM00969 (normal) & 20 & 2 & $0.834 \pm 0.04$ & $2.1 \pm 1.8$ \\
AG09602 (normal) & 20 & 92 & $0.825 \pm 0.06$ & $7.2 \pm 9.6$ \\
AG11513 (HGPS) & 8 & 8 & $0.836 \pm 0.08$ & $2.7 \pm 0.01$ \\
AG11513 (HGPS) & 18 & 8 & $0.776 \pm 0.10$ & $23.8 \pm 2.1^{\mathrm{a}}$ \\
AG11513 + SIRT6 (HGPS) & 22 & 8 & $0.829 \pm 0.06$ & $7.4 \pm 2.0^{\mathrm{b}}$ \\
\hline
\end{tabular}

0.04; old normal donor: $0.825 \pm 0.06$; HGPS PD 8: 0.836 \pm 0.08 ; HGPS PD 18: $0.776 \pm 0.1)$ or HGPS cells with ectopic SIRT6 $(0.829 \pm 0.06)$.

It is generally believed that the accumulation of DNA damage is a critical step leading to the premature aging of HGPS cells [30, 31]. HGPS cells accumulate DNA damage, in particular double-strand breaks, with continued in vitro passaging $[30,31]$. Here, we have immunolabeled for 53BP1, an important regulator of the cellular response to double-strand breaks [32], in order to quantitatively compare the numbers of DNA damage foci in HGPS cells plus or minus ectopic SIRT6. We showed that parental HGPS cells exhibited numerous 53BP1positive DNA damage foci (fig. 6a, c) and following the stable expression of SIRT6 there was a significant reduction in the mean number of DNA damage foci per nuclei (fig. 6b, c).

\section{Discussion}

Here we have shown for the first time that HGPS cells, which are characterized by limited proliferative capabilities, genomic instability and premature aging $[4,5,22,27$, 28], display markedly reduced levels of SIRT6 protein compared to normal cells at a similar PD of about 15 . Of note, Mao et al. [33] have recently reported diminished levels of SIRT6 in primary cultures of normal human fibroblasts approaching replicative senescence, providing another example of the similarities between the aging of normal cells and premature cellular aging in the context of progeria. The signaling pathways triggering reduced SIRT6 in normal and HGPS cells remain unknown. We did not observe reduced SIRT6 in atypical HGPS cells (lacking progerin), prompting us to test whether progerin directly causes reduced SIRT6 in human cells. The fact that in vivo aged tissues and cells express progerin $[18,19$, 

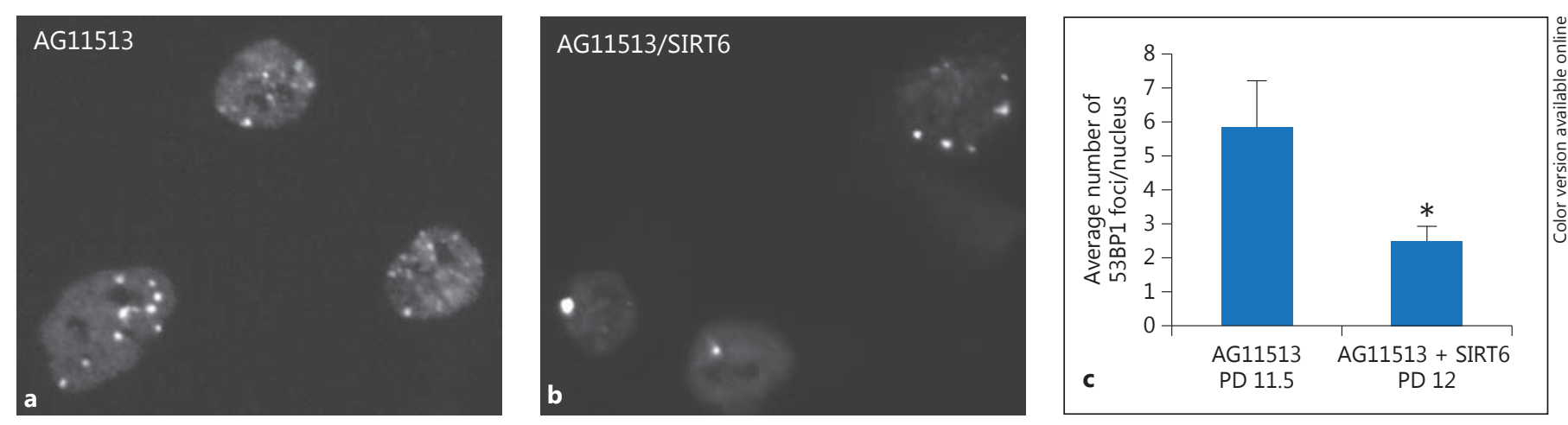

Fig. 6. Ectopic expression of SIRT6 is associated with a significant reduction in the number of 53BP1-positive DNA damage foci. Representative immunolabeling for 53BP1 in AG11513 HGPS cells in the absence (a) versus presence (b) of ectopic SIRT6. Images were captured with $\mathrm{a} \times 40$ objective. $\mathrm{c}$ Bars represent the fre-

quency of 53BP1 foci per nucleus in AG11513 fibroblasts \pm SIRT6. Data were derived from three independent experiments. Error bars represent the standard error of replicates. The asterisk denotes a statistically significant difference $(\mathrm{p} \leq 0.05)$.

34] makes this an intriguing hypothesis with potential implications for physiologic and premature aging processes. Recognizing that the progerin transcript and protein are expressed in skin biopsies of older individuals [34], we were initially surprised by our inability to detect progerin in primary fibroblasts from a 92-year-old donor by Western blotting. Since the levels of progerin are much lower in normal cells and tissues compared to those from HGPS donors [18, 19,34], this likely reflects our use of whole cell lysates rather than preparations that involved the extraction of soluble proteins and enrichment for membrane-associated proteins [18].

To test the hypothesis that progerin directly causes reduced SIRT6 expression, we relied on a tightly regulated, tetracycline-inducible system in hTERT-immortalized human fibroblasts. Our results were definitive: we did not detect any change in the levels of SIRT6 protein for up to 12 days of sustained, high expression of ectopic progerin. We therefore conclude that reduced SIRT6 is: (1) indirectly mediated by progerin but requires a more protracted time period, or (2) not related to progerin at all, but perhaps instead reflective of the near-senescent state of HGPS cells. Our qRT-PCR data revealed that typical HGPS cells display a marked reduction in SIRT6 mRNA, hinting that diminished levels of this sirtuin are at least partially mediated at the transcriptional level [unpubl. data]. This preliminary finding is unique from experimental models of nutrient bioavailability where SIRT6 is predominantly regulated at the level of protein stability [35]. It is well established that the accumulation of progerin causes structural defects in the nuclear lamina, an in-

terwoven network of A- and B-type lamins that not only serves as a shield to protect from mechanical stress but also plays a role in the regulation of gene expression [5, 18]. Therefore, it is plausible that progressive progerinmediated aberrations in nuclear architecture indirectly result in reduced transcription of the SIRT6 gene and/or alterations in the expression of genes regulating SIRT6 protein stability.

To date, the cellular consequences of reduced SIRT6 in HGPS cells are unclear, but our 53BP1 immunolabeling results preliminarily suggest that a reduction of this sirtuin impedes the repair of damaged DNA. As previously reported [36], we have shown that HGPS cells exhibit numerous DNA damage foci upon serial passaging in vitro. Moreover, our findings that restoration of SIRT6 protein levels is associated with increased cellular grow rates, reduced frequency of senescence and reduced numbers of 53BP1-positive DNA damage foci are collectively consistent with SIRT6 facilitating DNA repair in HGPS cells, a role that has been previously reported for SIRT6 in other cell types and experimental systems [12, 13, 15, 16, 37, 38].

Recognizing the involvement of SIRT6 in maintaining telomeres [15, 16], reduced expression of SIRT6 in HGPS could lead to less SIRT6 binding at telomeres, loss of telomeric heterochromatin, transcriptional activation of proximal genes (i.e. the telomere positional effect) [39], and/or telomere dysfunction. In turn, loss of heterochromatic marks, which may regulate telomere length [40], could contribute to telomere dysfunction and the induction of cellular senescence [40]. In mouse cells, SIRT6 interacts with NFKB, a stress-responsive transcription fac- 
tor, coregulating numerous genes involved in cellular senescence and organismal aging [41]. Interestingly, when SIRT6 is absent in murine cells, the coordinated regulation among NFkB target genes in response to stress (a defining characteristic of aging tissues) goes awry [42]. Perhaps reduced SIRT6 contributes to elevated levels of NFkB activation in HGPS cells [43], which appears to be mechanistically linked to the induction of cellular senescence [44].

Regardless of the underlying molecular mechanism leading to reduced SIRT6 in HGPS cells and the numerous possible cellular consequences that may follow, we have demonstrated that restoring SIRT6 expression in typical HGPS cells confers antiaging effects. Our repeated inability to expand HGPS cells following infection with 'empty vector' viral supernatant and puromycin selection, coupled with the ease of expanding drug-resistant HGPS cells ectopically expressing SIRT6, revealed that restored expression of this sirtuin provides a growth advantage to progeria cells. Ectopic SIRT6 expression was also associated with reduced PD times, reduced frequency of cellular senescence, fewer DNA damage foci and, consequently, an extension of the in vitro life span of HGPS cells. In further support of SIRT6 imparting antiaging effects, we have demonstrated that HGPS/SIRT6 cells display a significantly reduced frequency of abnormal nuclei compared to uninfected controls. Future investigations are needed to determine whether SIRT6-mediated improvements in the nuclear architecture of HGPS cells correct the recognized alterations in mechanical properties and gene expression [45]. In support of this latter possibility, Scaffidi and Misteli [22] have previously shown that reversal of the severe morphological abnormalities of the nuclear envelope by correcting for the aberrant LMNA splicing event in HGPS cells did restore the expression of many misregulated genes. There is no doubt that the mechanistic relationship between nuclear atypia and the induction of senescence in HGPS cells is complex, as is the manner in which ectopic SIRT6 rescues age-associated phenotypes.

Traditionally, it has been assumed that due to the dominant negative nature of mutant lamin A protein, reversal of the cellular phenotypes in HGPS cells requires the elimination of the mutant protein [22]. The data presented here challenges this paradigm by demonstrating that aging-associated phenotypes in progerin-expressing HGPS cells can be improved by restoring expression of a single epigenetic modulator. Additional investigations are now warranted to define: (1) the extent and duration of these phenotypic improvements; (2) the functional do- mains and enzymatic activities of SIRT6 required for conferring anti-aging effects in HGPS cells, and (3) the pathways contributing to the reduction in SIRT6 expression in HGPS cells as well as to the improved age-related phenotypes upon restoration of SIRT6.

\section{Acknowledgements}

The author(s) acknowledge receipt of the following financial support for the research, authorship, and/or publication of this article: VCU Massey Cancer Center National Cancer Institute Training Grant (T32) in Cancer Biology (J.M.-S.) and VCU Department of Pathology (L.E.). The microscopy was performed at the Virginia Commonwealth University Department of Anatomy and Neurobiology Microscopy Facility, supported in part with funding from NIH-NINDS Center Core Grant (5P3ONSO47463-02). Viral services and products in support of this investigation were generated by the VCU Massey Cancer Center Biological Macromolecule Shared Resource, supported in part with funding from NIH-NCI Cancer Center Support Grant (P30 CA016059) and in part by the Intramural Research Program of the $\mathrm{NIH}$, National Cancer Institute, Center for Cancer Research. We are grateful to Dr. Colleen Jackson-Cook for use of the Bioview Duet Scanning Station for obtaining images for the determination of contour ratios.

\section{Disclosure Statement}

There is no conflict of interest to disclose.

References

1 Serio R: Unraveling the mysteries of aging through a Hutchinson-Gilford progeria syndrome model. Rejuvenation Res 2011;14: 133-141.

-2 Eriksson M, Brown W, Gordon L, Glynn M, Singer J, Scott L, Erdos M, Robbins C, Moses $\mathrm{T}$, Berglund $\mathrm{P}$, et al: Recurrent de novo point mutations in lamin A cause Hutchinson-Gilford progeria syndrome. Nature 2003;423: 293-298.

3 D’Apice R, Tenconi R, Mammi I, van den Ende J, Novelli G: Paternal origin of LMNA mutations in Hutchinson-Gilford progeria. Clin Genet 2004;65:52-54.

4 Benson E, Lee S, Aaronson S: Role of progerin-induced telomere dysfunction in HGPS premature cellular senescence. J Cell Sci 2010; 123:2605-2612.

5 Cao K, Blair C, Faddah D, Kieckhaefer J, Olive M, Erdos M, Nabel E, Collins F: Progerin and telomere dysfunction collaborate to trigger cellular senescence in normal human fibroblasts. J Clin Invest 2011;121:2833-2844.

-6 Scaffidi P, Gordon L, Misteli T: The cell nucleus and aging: tantalizing clues and hopeful promises. PLoS Biol 2005;3:e395. 
7 Berdasco M, Esteller M: Hot topics in epigenetic mechanisms of aging. Aging Cell 2012; 11:181-186.

8 Arancio W, Pizzolanti G, Genovese S, Pitrone $\mathrm{M}$, Giordano C: Epigenetic involvement in Hutchinson-Gilford progeria syndrome: a mini-review. Gerontology 2014;60:197-203.

9 Bitterman K, Medvedik O, Sinclair D: Longevity regulation in Saccharomyces cerevisiae: linking metabolism, genome stability, and heterochromatin. Microbiol Mol Biol Rev 2003;67:376-399.

10 Chen D, Guarente L: SIR2: a potential target for calorie restriction mimetics. Trends $\mathrm{Mol}$ Med 2007;13:64-71.

11 Carafa V, Nebbioso A, Altucci L: Sirtuins and disease: the road ahead. Front Pharmaco 2012;3:4.

12 Kugel S, Mostoslavsky R: Chromatin and beyond: the multitasking roles of SIRT6. Trends Biochem Sci 2014;29:72-81.

13 Mostoslavsky R, Chua K, Lombard D, Pang W, Fischer M, Gellon L, Liu P, Mostoslavsky G, Franco S, Murphy M, et al: Genomic instability and aging-like phenotype in the absence of mammalian SIRT6. Cell 2006;124:315329.

14 Kanfi Y, Naiman S, Amir G, Peshti V, Zinman G, Nahum L, Bar-Joseph Z, Cohen H: The sirtuin SIRT6 regulates lifespan in male mice. Nature 2012;483:218-221.

15 Jia G, Su L, Singhal S, Liu X: Emerging roles of SIRT6 on telomere maintenance, DNA repair, metabolism and mammalian aging. Mol Cell Biochem 2012;364:345-350.

-16 Tennen R, Chua K: Chromatin regulation and genome maintenance by mammalian SIRT6. Trends Biochem Sci 2011;36:39-46.

-17 Michishita E, McCord R, Berber E, Kioi M, Padilla-Nash H, Damian M, Cheung P, Kusumoto R, Karahara T, Barrett J, et al: SIRT6 is a histone $\mathrm{H} 3$ lysine 9 deacetylase that modulates telomeric chromatin. Nature 2008;52: 492-496.

18 Scaffidi P, Misteli T: Lamin A-dependent nuclear defects in human aging. Science 2006; 312:1059-1063.

19 Olive M, Harten I, Mitchell R, Beers J, Djabali K, Cao K, Erdos M, Blair C, Funke B, Smoot $\mathrm{L}$, et al: Cardiovascular pathology in Hutchinson-Gilford progeria: correlation with the vascular pathology of aging. Arterioscler Thromb Vasc Biol 2010;30:2301-2309.

20 Scaffidi P, Misteli T: Lamin A dependent misregulation of adult stem cells associated with accelerated aging. Nat Cell Biol 2008;10:452459.
21 Kandert S, Lüke Y, Kleinhenz T, Neumann S, Lu W, Jeagar V, Munck M, Wehnert $M$, Muller C, Zhou Z, et al: Nesprin-2 giant safeguards nuclear envelope architecture in LMNA S143F progeria cells. Hum Mol Genet 2007;16:2944-2959.

22 Scaffidi P, Misteli T: Reversal of the cellular phenotype in the premature aging disease Hutchinson-Gilford progeria syndrome. Nat Med 2005;11:440-445.

23 Elmore L, Rehder C, Di X, McChesney P, Jackson-Cook C, Gewirtz D, Holt S: Adriamycin-induced senescence in breast tumor cells involves functional p53 and telomere dysfunction. J Biol Chem 2002;277:3550935515.

24 Liang L, Zhang H, Gu X: Homozygous LMNA mutation R527C in atypical Hutchinson-Gilford progeria syndrome: evidence for autosomal recessive inheritance. Acta Paediatr 2009; 98:1365-1368.

25 Garg A, Subramanyam L, Agarwal A, Simha V, Levine B, D'Apice M, Novelli G, Crow Y: Atypical progeroid syndrome due to heterozygous missense LMNA mutations. J Clin Endocrinol Metab 2009;94:4971-4983.

26 Ardestani P, Liang F: Sub-cellular localization, expression and functions of Sirt6 during the cell cycle in HeLa cells. Nucleus 2012;3: 442-451.

27 Ouellette M, McDaniel L, Wright W, Shay J, Schultz R: The establishment of telomeraseimmortalized cell lines representing human chromosome instability syndromes. Hum Mol Genet 2000;9:403-411.

28 Tivey H, Brook A, Rokicki M, Kipling D, Davis T: p38 (MAPK) stress signaling in replicative senescence in fibroblasts from progeroid and genomic instability syndromes. Biogerontology 2013;14:47-62.

29 Goldman R, Shumaker D, Erdos M, Eriksson M, Goldman A, Gordon L, Gruenbaum Y, Khuon S, Mendez M, Varga R, Collins F: Accumulation of mutant lamin A causes progressive changes in nuclear architecture in Hutchinson-Gilford progeria syndrome. Proc Natl Acad Sci USA 2004;101:8963-8968.

30 Liu Y, Rusinol A, Sinensky M, Wang Y, Zou A: DNA damage responses in progeroid syndromes arising from defective maturation of prelamin A. J Cell Sci 2006;2119:4644-4649.

31 Musich P, Zou Y: Genomic instability and DNA damage responses in progeria arising from defective maturation of prelamin A. Aging 2009;1:28-37.

32 Panier S, Boulton SJ: Double-strand break repair: 53BP1 comes into focus. Nat Rev Mol Cell Biol 2014;15:7-18.
33 Mao Z, Tian X, van Meter M, Ke Z, Gorbunova V, Seluanov A: Sirtuin 6 (SIRT6) rescues the decline of homologous recombination repair during replicative senescence. Proc Natl Acad Sci USA 2012;109;11800-11805.

34 McClintock D, Ratner D, Lokuge M, Owens D, Gordon L, Collins F, Djabali K: The mutant form of lamin A that causes Hutchinson-Gilford progeria is a biomarker of cellular aging in human skin. PLoS One 2007;2:e1269.

35 Kanfi Y, Shalman R, Peshtia V, Pilosofa S, Gozlana, Y, Pearsonb K, Lerrera B, Moazedc D, Marined J-C, Cabob R, et al: Regulation of SIRT6 protein levels by nutrient availability. FEBS Lett 2008;582:543-548.

36 Liu B, Wang J, Chan KM, Tija WM, et al: Genomic instability in laminopathy-based premature aging. Nat Med 2005;11:780-785.

- 37 McCord R, Michishita E, Hong T, Berber E, Boxer L, Kusumoto R, Guan S, Shi X, Gozani O, Burlingame A, Bohr V, Chua K: SIRT6 stabilizes DNA-dependent protein kinase at chromatin for DNA double-strand break repair. Aging 2009;1:109-121.

- 38 Lombard D, Schwer B, Alt F, Mostoslavsky R SIRT6 in DNA repair, metabolism, and ageing. J Intern Med 2008;263:128-141.

39 Tennen R, Bua D, Wright W, Chua K: SIRT6 is required for the maintenance of telomere position effect in human cells. Nat Commun 2011;2:433.

40 DiMauro T, David G: Chromatin modification: the driving force of senescence and aging? Aging 2009;1:182-190.

-41 Hayden MS, Ghosh S: Shared principles in NF- $\kappa B$ signaling. Cell 2008;132:344-362.

42 Kawahara T, Rapicavoli N, Wu A, Qu K, Quake S, Chang H: Dynamic chromatin localization of Sirt6 shapes stress- and aging-related transcriptional networks. PLoS Genet 2011;7:e1002153.

43 Adler A, Sinha S, Kawahara T, Zhang J, Segal E, Chang H: Motif module map reveals enforcement of aging by continual NF- $\mathrm{kB}$ activity. Genes Dev 2007;21:3244-3257.

44 Tilstra J, Robinson A, Wang J, Gregg S, Clauson C, Reay D, Nasto L, St Croix C, Usas A, Vo N, et al: NF- $\kappa B$ inhibition delays DNA damage-induced senescence and aging in mice. J Clin Invest 2012;122:2601-2612.

45 Dahl K, Scaffidi P, Islam M, Yodh A, Wilson K, Misteli T: Distinct structural and mechanical properties of the nuclear lamina in Hutchinson-Gilford progeria syndrome. Proc Natl Acad Sci USA 2006;103:10271-10276. 